\title{
AUTHOR PREPARED MANUSCRIPTS IN COMPUTER-READABLE FORM FOR POWDER DIFFRACTION
}

The business of publishing scientific articles is evolving rapidly to keep up with modern technological developments. I have long recognized that the principal role of the computer to mankind will be not to enhance the activities of elaborate computational science but rather to promote the "Age of Communication." The beginnings of that age are with us now, and we need to start taking advantage of the virtues of the new technology.

It is now possible for the author to compose the manuscript in his/her private office in digital form and have the manuscript go directly to the publishers for final composition without intervening resetting. This possibility eliminates the potential introduction of errors during the composing stages which is especially valuable in the preparation of tables involving numbers that may have initially been computer generated. Everyone in the publishing business knows the difficulty of detecting such errors once they occur in manuscript preparation.

In order to take full advantage of the possibilities of electronic publishing of technical articles, authors need to follow the procedures established by the publishers. Powder Diffraction is published by the American Institute of Physics, AIP, leader in the implementation of new methodology. The AIP is now accepting computer manuscripts for use in production if the author has used certain acceptable manuscript composition programs. The acceptable programs are REVTeX, LaTeX, Word, and WordPerfect. Files from these programs are directly translatable into the AIP Xyvision internal composition system. In addition to the accuracy of table conversion, equations are directly readable. One very powerful advantage of LaTeX is that it is also easy to mail the file electronically because the nonprinting control strings are imbedded in the text in a manner that does not interfere with the transmission of the file.

Authors interested in participating in this program for Powder Diffraction manuscripts should indicate so in their covering letters when submitting the original manuscript to the Powder Diffraction editor for technical review. This opportunity does not replace the requirement that three printed copies are neces- sary with the first submission for the purposes of the technical review and acceptance. It is very rare for any manuscript to pass through this review without many recommended revisions for manuscript modification, so co-submission of a computerreadable disk at initial submission time is not recommended. Authors will be advised of the appropriate time to prepare the final version for transfer to the publication office. If you already have a manuscript in the review stage, you will be advised of the submission options when you receive the results of the technical review.

AIP will be using internal software to convert the acceptable files to the format required by the internal composition system. For this project to benefit both the author and the AIP production effort, it is essential that, if you are using Word or WordPerfect, the Word/WordPerfect Author Toolkit is used to typeset the final version of the manuscript. Also, the files should be saved as Word or WordPerfect files. Do not save the files in ASCII format. If you are using REVTeX, it is imperative that the guidelines as documented in the REVTeX toolbox are followed precisely. Each submitted file will be evaluated for its suitability; authors will receive notice with their galley proof stating whether or not their electronic file was used.

The REVTeX toolbox is available via anonymous FTP on the Internet from ftp.aip.org in the directory/pub/revtex. The Word/WordPerfect Author Toolkit may be found in the directory /ems. The macros and the Toolkit are also available by accessing the AIP HomePage (http://www.aip.org/epub/ compuscripts.info.html). If you wish to receive the macros or Toolkit on a diskette, please contact Katie Bell (e-mail: katie@aip.org; phone; 516-576-2286). Please specify DOS or Mac diskette. If you have any further questions, please contact Liz Belmont (esubs@aip.org).

Deane K. Smith

Editor-in-Chief

with considerable assistance from the staff at AIP 